- RAM, REV. ADM. MACKENZIE, 16(6), Edição Especial • SÃO PAULO, SP • NOV./DEZ. 2015 • ISSN 1518-6776 (impresso) • ISSN 1678-6971 (on-line) • http://dx.doi.org/10.1590/1678-69712015/administracao.v16n6p71-93. Submissão: 1 jul. 2014. Aceitação: 25 ago. 2015. Sistema de avaliação: às cegas dupla (double blind review). UNIVERSIDADE PRESBITERIANA MACKENZIE. Silvio Popadiuk (Ed.), Eda Castro Lucas de Souza (Ed. Seção), p. 71-93.

\title{
tis BEM-ESTAR NO TRABALHO E PERCEPÇÃO DE SUCESSO NA CARREIRA COMO ANTECEDENTES DE INTENÇÃO DE ROTATIVIDADE
}

PAULA RODRIGUES AGAPITO

Mestra em Administração pelo Programa de Pós-Graduação em Administração da Universidade Metodista de São Paulo (Umesp).

Professora convidada do Centro de Ciências Sociais Aplicadas da Universidade Presbiteriana Mackenzie (CCSA-UPM). Rua da Consolação, 930, Prédio T, Térreo, Higienópolis, São Paulo - SP - Brasil - CEP 01302-907

E-mail: paula.agapito1@yahoo.com.br

\section{ANGELO POLIZZI FILHO}

Mestre em Administração pelo Programa de Pós-Graduação em Administração da Universidade Metodista de São Paulo (Umesp). Professor do Departamento de Ciências Gerenciais da Universidade Nove de Julho (Uninove). Avenida Professor Luiz Ignácio de Anhaia Mello, 1.363, Vila Prudente, São Paulo - SP - Brasil - CEP 03132-100 E-mail:profapolizzi@uol.com.br

\section{MIRLENE MARIA MATIAS SIQUEIRA}

Doutora em Psicologia pela Universidade de Brasília (UnB).

Professora (aposentada) do Instituto de Psicologia da Universidade Federal de Uberlândia (UFU).

Avenida Pará, 1.720, Umuarama, Uberlândia - MG - Brasil - CEP 38400-902

E-mail: mirlenesiqueira@uol.com.br

Este artigo pode ser copiado, distribuído, exibido, transmitido ou adaptado desde que citados, de forma clara e explícita, o nome da revista, a edição, o ano e as páginas nas quais o artigo foi publicado originalmente, mas sem sugerir que a RAM endosse a reutilização do artigo. Esse termo de licenciamento deve ser explicitado para os casos de reutilização ou distribuição para terceiros. Não é permitido o uso para fins comerciais. 


\section{RESUMO}

O objetivo geral deste estudo foi analisar, interpretar e discutir as relações entre as percepções de sucesso na carreira, o bem-estar no trabalho e a intenção de rotatividade em trabalhadores da Região Sudeste do Brasil. Participaram desta pesquisa 500 trabalhadores que atuam no Estado de São Paulo, em organizações não governamentais, públicas e privadas. Como instrumento para coleta de dados foi utilizado um questionário de autopreenchimento composto de cinco escalas que mediram as variáveis da pesquisa. A presente pesquisa se propôs a apresentar, interpretar e discutir as relações entre as variáveis, como também testar as hipóteses referentes ao modelo conceitual proposto, por meio de uma pesquisa de natureza transversal com abordagem quantitativa, cujos dados coletados foram analisados por aplicação de técnicas estatísticas paramétricas (cálculos de estatísticas descritivas: médias, desvio padrão, teste $t$ e correlações; cálculos de estatísticas multivariadas: análise de regressão linear múltipla stepwise e teste da normalidade das variáveis, por meio do teste de Kolmogorov-Smirnov). O tratamento e a análise dos dados foram realizados pelo software estatístico Statistical Package for the Social Science (SPSS), versão I8.o. Os resultados obtidos demonstraram que as dimensões de bem-estar no trabalho exercem forte e significativo impacto sobre a intenção de rotatividade dos profissionais, enquanto a percepção de sucesso na carreira contribuiu com valores baixos nesse impacto, devido ao formato do modelo hipotético. A pesquisa possibilitou concluir que quanto mais a empresa se preocupa em proporcionar um ambiente de trabalho que seja animador, interessante e que cause entusiasmo, menos os profissionais pensarão em deixá-la.

\section{PALAVRAS-CHAVE}

Comportamento organizacional. Bem-estar no trabalho. Percepção de sucesso na carreira. Carreira. Rotatividade. 


\section{INTRODUÇ ÃO}

Pessoas sempre foram fundamentais para as organizações, mas a importância estratégica delas está crescendo. Uma nova geração de estratégias competitivas está surgindo, e conceitos como vantagem competitiva, cadeia de valor, orientação para o crescimento, entre outros, passaram a ser repensados e alinhados às teorias mais recentes (Busbin, Johnson, \& DeConinck, 2008). Pode-se dizer que as pessoas que constituem uma organização são uma fonte valiosa de vantagem competitiva. Se a empresa souber aproveitar e desenvolver as qualidades e os conhecimentos específicos desses indivíduos, ela obterá eficácia nos produtos e serviços, e, consequentemente, um diferencial competitivo em relação aos concorrentes.

No passado, muitos estudiosos chegaram a apontar que as máquinas eliminariam o trabalho humano, entretanto hoje ocorre o oposto: as pessoas nunca foram tão importantes nas empresas. Em muitas economias que apresentam rápido crescimento, pode ser mais fácil ter acesso ao dinheiro e à tecnologia do que a bons profissionais (Gubman, I998).

Diante desse cenário, surgiu o interesse dos pesquisadores pelas emoções decorrentes do contexto do trabalho, tendo um grande aumento de investigações sobre conceitos afetivos, tais como afetos positivos e negativos, estados de ânimo e emoções que envolvem as experiências emocionais no contexto de trabalho, levando à compreensão de fenômenos como estresse, burnout, sofrimento e saúde mental dos trabalhadores (Brief \& Weiss, 2002; Gondim \& Siqueira, 2004).

E para que as organizações contem com uma força de trabalho preparada para assumir diferentes tarefas nos mais variados contextos organizacional, devem preocupar-se com o bem-estar dos profissionais (Siqueira \& Gomide, 2004), obtendo melhores avaliações em relação à chefia, aos colegas, ao salário, às oportunidades de promoções e à natureza do trabalho (Siqueira, 2008; Siqueira \& Padovam, 2008; Tamayo, 2000).

Segundo Paschoal e Tamayo (2008), o bem-estar no trabalho pode ser conceituado como a prevalência de emoções positivas na empresa e a percepção do indivíduo de que nela poderá expressar e desenvolver os próprios potenciais, avançar nas metas de vida e reduzir uma possível intenção de sair da organização (Davis \& Newstrom, 2002). Além disso, o fato de o funcionário ter com a empresa uma percepção positiva que satisfaça os interesses de carreira dele pode levá-lo a apresentar melhores resultados para a organização (Maynard \& Joseph, 2008). De acordo com Costa (20II), carreira é a sequência de posições ocupadas durante a vida de uma pessoa, em função de percepções individuais, atitudes e 
comportamentos profissionais que resultam em desenvolvimento de competências para lidar com situações de trabalho de maior complexidade e em constante transformação.

Tendo esse contexto como pano de fundo, a proposta deste estudo é investigar o impacto das percepções de sucesso na carreira e no bem-estar no trabalho, na intenção de rotatividade nas organizações. Acredita-se que essas variáveis do comportamento organizacional, se utilizadas como ferramentas de gestão, auxiliarão na satisfação dos profissionais com a modalidade de trabalho e as suas perspectivas de carreira, além de possibilitarem uma redução da rotatividade, de modo que o funcionário se sinta parte integrante do contexto social em que atua.

\section{BEM-ESTAR NO TRABALHO}

Desde I984, Diener e Ryff pesquisam aspectos relacionados ao bem-estar dos indivíduos. Outra vertente surgiu em I998 com Keyes, que buscou analisar o funcionamento das relações do indivíduo no contexto social no qual está inserido. No entanto, os estudos que buscavam a compreensão sobre questões subjetivas, psicológicas e sociais não foram suficientes para a compreensão desses indivíduos no ambiente de trabalho.

Diversas escalas têm sido elaboradas no Brasil e no exterior, as quais se propõem a medir o bem-estar no trabalho, conforme os estudos de Watson, Clark e Tellegen (I988), Daniels (2000), Van Horn, Taris, Schaufeli e Scheurs (2004), Albuquerque e Tróccoli (2004), Paschoal e Tamayo (2008) e Dessen e Paz (20I0). Entretanto, para a realização desta pesquisa, utilizou-se o modelo teórico de bem-estar no trabalho (BET) desenvolvido por Siqueira e Padovam (2008), variável composta por três dimensões: satisfação no trabalho, envolvimento com o trabalho e comprometimento organizacional afetivo.

Segundo Siqueira e Gomide (2004), essas três dimensões podem representar tanto os vínculos positivos com o trabalho quanto com a organização, representado por comprometimento organizacional afetivo. A dimensão denominada satisfação no trabalho pode ser definida como o grau de contentamento com relacionamentos no trabalho, chefias e colegas, trabalho realizado e sistema de recompensas, envolvendo salários e promoções. O envolvimento com o trabalho é o nível de identificação com o trabalho realizado, e o comprometimento organizacional afetivo refere-se aos sentimentos positivos e negativos que o empregado poderia ter em relação à organização empregadora (Siqueira, 2008). O modelo pode ser mais bem compreendido por meio da Figura I. 


\section{FIGURA I}

\section{MODELO TEÓRICO DE BEM-ESTAR NO TRABALHO (BET)}

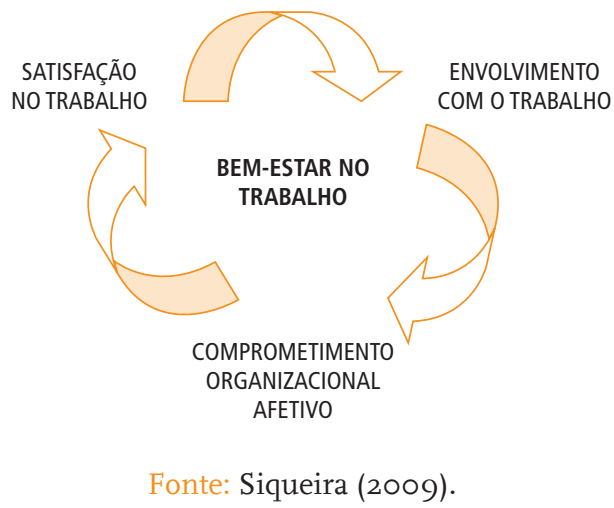

Siqueira e Gomide (2004) definem satisfação no trabalho como um vínculo afetivo positivo com a empresa que engloba satisfação com chefias, colegas, salário, oportunidades de promoção e tarefas realizadas. Por esse motivo, torna-se evidente e relevante esse construto fazer parte do modelo proposto pelas autoras, uma vez que engloba aspectos concernentes a todos os tipos de organizações, não somente permitindo analisar comportamentos de profissionais que convivem com esses fatores no dia a dia, mas também daqueles que sofrem pela ausência de algum deles.

Envolvimento com o trabalho, a segunda dimensão de BET, foi um conceito criado inicialmente por Lodhal e Kejner (i965, p. 25) como "o grau em que o desempenho de uma pessoa no trabalho afeta sua autoestima”. Segundo Siqueira e Padovam (2008), esse conceito tem relação com a ideia de fluxo criada por Csikszentmihalyi (I999), que define esse estado como uma sensação de ação sem esforço, como as que ocorrem nos melhores momentos de vida do indivíduo, ou seja, as habilidades totalmente voltadas para a superação de um desafio que está no limite da capacidade de controle. Geralmente, essas experiências envolvem um equilíbrio entre a capacidade de o indivíduo agir e as oportunidades disponíveis para a ação. Entende-se que o trabalho pode ser uma oportunidade para oferecer condições para que o indivíduo entre em fluxo (Csikszentmihalyi, 2003). Para Siqueira e Gomide (2004), os fatores pessoais são fundamentais para a explicação do envolvimento com o trabalho e delegação de funções realizada pela chefia. Com base nesses fatores, pode-se oferecer um melhor tratamento aos trabalhadores e aperfeiçoar o diálogo com eles.

A terceira dimensão de BET, o comprometimento organizacional afetivo, é apresentada como "um estado no qual um indivíduo se identifica com uma 
organização particular em seus objetivos" (Mowday, Steers, \& Porter, I979, p. 225). De acordo com Siqueira e Padovam (2008), essa dimensão não está ligada diretamente ao trabalho, mas à organização, pois se considera a ligação positiva que o profissional tem com a organização, e não com o trabalho em si. Para as autoras, o profissional comprometido afetivamente acredita que esse sentimento pode refletir em vivências positivas e prazerosas.

\section{INTENÇÃO DE ROTATIVIDADE}

Rotatividade de pessoal (turnover) corresponde à proporção de empregados que deixam a organização em um determinado período. Os estudos sobre rotatividade surgiram com Brayfield e Crockett (I955) e Herzberg, Mausner, Peterson e Capwell (I959) que encontraram fortes evidências na relação entre turnover e absenteísmo. Brayfield e Crockett (I955) foram além ao apontarem fragilidades metodológicas em uma série de estudos, como o fracasso em obter medidas e o uso de ferramentas de medição fracas ou ambíguas que sinalizavam uma necessidade de maior rigor em técnicas de pesquisa. Vroom (i964) também foi um dos primeiros a apresentar uma relação negativa consistente entre a satisfação no trabalho e a intenção de rotatividade, confirmada até a atualidade pelas diversas pesquisas realizadas (Campos \& Malik, 2008; Medeiros et al., 20Io; Menezes \& Bastos, 20ıо; Cavazotte, Oliveira, \& Miranda, 20ıо).

Neste trabalho, o interesse é saber quais são as razões que levam as pessoas a deixar as empresas em que trabalham, uma vez que a perda de bons empregados afeta diretamente a eficiência das organizações e pode comprometer as estratégias em busca da competitividade (Mintzberg \& Lampel, I999). Entretanto, "conhecer os motivos que levam as pessoas a deixar as organizações onde trabalham e reduzir as taxas de turnover se tornam desafios importantes no gerenciamento das organizações que buscam tanto a eficiência como a eficácia organizacional" (Ferreira, 200I, p. I6).

A intenção de rotatividade é um construto que mede a intenção de um profissional de sair do emprego atual, e os resultados dessa medida ajudam os gestores a desenvolver ações capazes de fomentar a permanência dos bons profissionais na organização. Dessa forma, diminuem-se os gastos com um novo processo de recrutamento e seleção, o departamento continua funcionando regularmente, evita-se que o profissional seja contratado pela concorrência, além de outros benefícios (Stewart, Volpone, Avery, \& McKay, 20II). Com base em estudos da área, Davis e Newstrom (2002) concluem que empregados satisfeitos estão menos propensos a pensar em pedir demissão e apresentam maior probabilidade de ficarem mais tempo na organização. 


\section{PERCEPÇÃO DE SUCESSO NA CARREIRA}

Atualmente, entre os trabalhos relevantes sobre organizações, encontra-se o estudo sobre carreiras que busca compreender o comportamento do trabalhador em relação à política de gestão de pessoas da organização e o grau de dependência dele em relação à própria empresa. Hughes (I937) definiu carreira como a forma de os indivíduos verem sentido em suas passagens pela vida, como também o que isso revela sobre a natureza e constituição do trabalho de uma sociedade.

Há muitos estudos que discutem o tema, que evoluiu desde a simples execução do trabalho, de modo servil, alienado e repetitivo, até um modelo presente nos dias de hoje, evidenciado pela construção ativa e independente da própria carreira por parte do funcionário, caso haja iniciativa e oportunidade por parte dele (DiMaggio \& Powell, ı983). Essas posições são influenciadas e negociadas considerando motivos e aspirações individuais, expectativas e imposições da organização e da sociedade (Hughes, I937; London \& Stumpf, I982; Dutra, 2002; Le Boterf, 2003; Motta, 2006).

Não é objetivo deste estudo analisar as carreiras, mas considerar a percepção dos profissionais sobre as próprias carreiras e verificar de que forma essa percepção pode influenciar a intenção deles de sair da organização em que trabalham.

Costa (2OII) tinha como objetivos: criar e validar um instrumento de mensuração da percepção de sucesso na carreira; definir os fatores que compõem essa escala de mensuração e verificar qual deles tem mais relevância; adaptar a escala de intenções comportamentais de comprometimento organizacional (Eicco) validada por Menezes (2009) para uma amostra de professores universitários; definir empiricamente que fatores compõem a Eicco e verificar quais têm mais relevância; realizar testes para averiguar se a percepção de sucesso na carreira explica significativamente as Icco; investigar que fatores da percepção de sucesso na carreira têm mais relação com as Icco. Os dados obtidos por Costa (20II) resultaram em uma escala de percepção de sucesso na carreira com 48 itens e dividida em II fatores, que se agrupam em duas dimensões denominadas objetivas e subjetivas.

A literatura revisada sobre bem-estar no trabalho, intenção de rotatividade e percepção de sucesso na carreira mostra que o bem-estar no trabalho é o tema mais antigo e mais investigado tanto no Brasil quanto no exterior. Por sua vez, a percepção de sucesso na carreira constitui-se em um construto novo que ainda requer dos pesquisadores maior atenção em futuros estudos.

Deve-se apontar também que, na literatura, não foram encontrados registros de estudos que investigaram, simultaneamente, os níveis de bem-estar vivenciados pelo indivíduo no ambiente organizacional, o plano de deixar a empresa no futuro e a percepção de sucesso na carreira. Dada essa lacuna, torna-se relevante o desenvolvimento do presente estudo cujos objetivos estão apresentados na sequência. 


\section{PROCEDIMENTOS METODOLÓGICOS}

A análise do referencial teórico direcionou suposições para a elaboração das seguintes hipóteses que envolvem os construtos do estudo:

- Hr: Percepção de sucesso na carreira tem um impacto significativo e negativo sobre intenção de rotatividade.

- H2: Bem-estar no trabalho tem um impacto significativo e negativo sobre intenção de rotatividade.

- Hza: Satisfação no trabalho tem um impacto significativo e negativo sobre intenção de rotatividade.

- H2b: Envolvimento com o trabalho tem um impacto significativo e negativo sobre intenção de rotatividade.

- H2c: Comprometimento organizacional afetivo tem um impacto significativo e negativo sobre intenção de rotatividade.

Dessa forma, com as hipóteses e os construtos definidos, o modelo conceitual do estudo pode ser visualizado na Figura 2.

\section{FIGURA 2}

\section{MODELO DE PERCEPÇÃO DE SUCESSO NA CARREIRA \\ E BEM-ESTAR NO TRABALHO COMO ANTECEDENTES \\ DE INTENÇÃO DE ROTATIVIDADE}

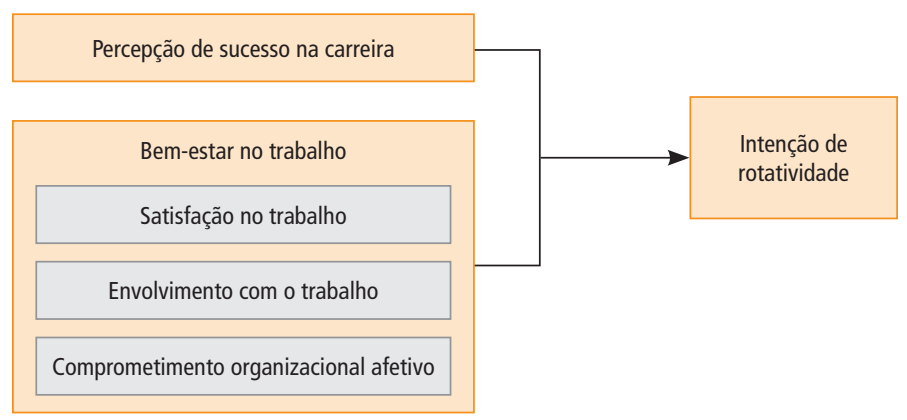

Fonte: Elaborada pelos autores.

\section{1 PARTICIPANTES}

A amostra foi constituída por 500 trabalhadores de empresas públicas e privadas localizadas no Estado de São Paulo, escolhidos por conveniência. Destes, $54,8 \%$ eram do sexo feminino. A média de idade era de 33 anos, e o estado civil 
predominante foi casado com $55 \%$. A escolaridade dos trabalhadores teve $30,8 \%$ com o nível médio completo, e o tempo médio de trabalho foi de 5,5 anos.

\subsection{INSTRUMENTO}

Para a coleta de dados, utilizou-se um questionário composto por cinco escalas que avaliaram as variáveis da pesquisa:

- Percepção de sucesso na carreira: construída e validada por Costa (20II), mede a percepção de sucesso na carreira sob a perspectiva do próprio indivíduo, independentemente do vínculo a uma organização em particular ou a várias organizações.

- Satisfação no trabalho: construída e validada por Siqueira (2008), contém, em sua forma reduzida, cinco fatores (satisfação com colegas de trabalho, satisfação com o salário, satisfação com a chefia, satisfação com a natureza do trabalho e satisfação com promoções), com três itens cada.

- Envolvimento com o trabalho: construída e validada por Lodhal e Kejner (I965), e validada para o Brasil por Siqueira (2008), contém, em sua forma reduzida, seis itens.

- Comprometimento organizacional afetivo: medida construída e validada por Siqueira (I995), contém, em sua forma reduzida, cinco itens.

- Intenção de rotatividade: construída e validada por Siqueira, Gomide, Moura e Marques (I997), é composta por três itens.

Os dados foram coletados por alunos do curso de Gestão de Recursos Humanos da modalidade a distância de uma universidade privada, como parte de um exercício avaliativo de conclusão do semestre letivo. Os questionários e o Termo de Consentimento Livre e Esclarecido (TCLE) foram enviados aos alunos por plataforma digital, para que eles pudessem imprimir e aplicar a pesquisa. O acesso ao campo de pesquisa foi facilitado pela utilização de uma carta de apresentação da universidade, contendo esclarecimentos sobre a finalidade e importância da pesquisa.

A devolução dos questionários e termos dos participantes foi realizada diretamente com o aluno responsável pela coleta dos dados em envelopes separados, e o envio para a pesquisadora responsável por esta pesquisa foi realizada por meio de malote, em envelopes igualmente separados.

\subsection{ANÁLISE DOS DADOS}

Os dados coletados formaram um banco de dados para tratamento no software estatístico Statistical Package for the Social Sciences (SPSS), versão I8.o. Realizou-se uma análise exploratória dos dados para verificar a precisão de entrada 
de dados, outliers e respostas omissas. Depois da exploração, análises descritivas foram realizadas a fim de caracterizar os participantes, assim como as correlações entre as variáveis ( $r$ de Pearson). Realizaram-se comparações entre as médias das variáveis e os pontos médios das respectivas escalas de medidas, assim como o cálculo de precisão das medidas. Após a exploração dos dados, efetuaram-se análises estatísticas multivariadas para teste das hipóteses, por meio de modelos de regressão linear múltipla e stepwise, utilizando o subprograma regression do SPSS, versão I8.o.

\section{RESULtAdos E Discussão}

A Tabela I contém as médias, os desvios padrão, a amplitude de respostas, os valores do teste $t$, os coeficientes de correlação ( $r$ de Pearson) entre as variáveis, além dos índices de precisão das escalas utilizadas no estudo. Na tabela, verifica-se também que os índices de precisão são todos satisfatórios, atingindo resultados superiores a 0,70 , valor considerado por Nunnally (I975) como ponto de corte para uma medida ser considerada confiável e precisa.

\section{TABELA I}

SUMÁRIO DAS ESTATÍSTICAS DESCRITIVAS, DOS VALORES DO TESTE T, DOS COEFICIENTES DE CORRELAÇÃO (R DE PEARSON) ENTRE AS VARIÁVEIS UTILIZADAS NO ESTUDO E DOS ÍNDICES DE PRECISÃO DAS ESCALAS $(\mathrm{N}=500)$

\begin{tabular}{|c|c|c|c|c|c|c|c|c|c|}
\hline VARIÁVEIS & MÉDIA & DP & $\begin{array}{c}\text { AMPLITUDE } \\
\text { DE } \\
\text { RESPOSTA }\end{array}$ & $\begin{array}{c}\text { VALORES } \\
\text { DO } \\
\text { TESTE } t\end{array}$ & 1 & 2 & 3 & 4 & 5 \\
\hline \multicolumn{10}{|l|}{ Bem-estar no trabalho } \\
\hline 1. Satisfação no trabalho (ST) & 4,32 & 0,94 & $1-7$ & $7,64^{* * *}$ & $(0,91)$ & & & & \\
\hline $\begin{array}{l}\text { 2. Envolvimento com o trabalho } \\
\text { (ET) }\end{array}$ & 3,5 & 1,44 & $1-7$ & $-7,76^{\star * *}$ & $0,571^{* * *}$ & $(0,86)$ & & & \\
\hline $\begin{array}{l}\text { 3. Comprometimento } \\
\text { organizacional afetivo (COA) }\end{array}$ & 3,13 & 0,92 & $1-5$ & $3,26^{* * *}$ & $0,770^{* * *}$ & $0,563^{* * *}$ & $(0,93)$ & & \\
\hline $\begin{array}{l}\text { Percepcão de sucesso na } \\
\text { carreira }\end{array}$ & 4,72 & 1,11 & $1-7$ & $14,64^{* * *}$ & $0,604^{* * *}$ & $0,572^{* * *}$ & $0,558^{* * *}$ & $(0,87)$ & \\
\hline Intenção de rotatividade & 2,68 & 1,17 & $1-5$ & $-5,99^{* * *}$ & $-0,626^{* * *}$ & $-0,510^{* * *}$ & $-0,650^{* * *}$ & $-0,450^{* * *}$ & $(0,93)$ \\
\hline
\end{tabular}

Índices de confiabilidade das escalas entre parênteses, na linha diagonal. $* * * \mathrm{p}<0,001$. 
Na Tabela I, observa-se que a dimensão satisfação no trabalho alcançou média significativamente superior (média $=4,32 ; \mathrm{DP}=0,94 ; t=7,64 ; \mathrm{p}<0,00 \mathrm{I}$ ) ao ponto médio da escala de respostas (valor $=4)$. A dimensão envolvimento com o trabalho alcançou média inferior (média $=3,5 ; \mathrm{DP}=\mathrm{I}, 44 ; t=-7,76 ; \mathrm{p}<0, \mathrm{O} \mathrm{I}$ ) ao ponto médio da escala de respostas (valor $=4$ ). A dimensão comprometimento organizacional afetivo alcançou média superior (média $=3, \mathrm{I} 3 ; \mathrm{DP}=0,92 ; t=3,26$;

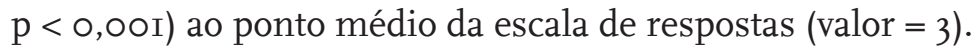

Esses dados possibilitam concluir que os participantes desta pesquisa apresentam resultados semelhantes aos diversos estudos já realizados que utilizaram as mesmas dimensões do bem-estar no trabalho e diferentes nas dimensões satisfação no trabalho (Siqueira \& Padovam, 2008) e comprometimento organizacional afetivo.

Quanto à percepção de sucesso na carreira, nota-se que essa variável alcançou média superior (média $=4,72$; DP $=\mathrm{I}, \mathrm{II} ; t=\mathrm{I} 4,64 ; \mathrm{p}<0, \mathrm{OOI}$ ) ao ponto médio da escala (valor $=4)$. Em relação à intenção de rotatividade, os resultados apresentaram média inferior (média $=2,68 ; \mathrm{DP}=\mathrm{I}, \mathrm{I} 7 ; t=-5,99 ; \mathrm{p}<0, \mathrm{O} \mathrm{I}$ ) ao ponto médio da escala de respostas (valor $=3$ ).

Com base nos resultados dos escores médios das dimensões de bem-estar no trabalho e da percepção de sucesso na carreira, observa-se que, apesar de os participantes terem um envolvimento relativamente baixo com o trabalho, apresentam satisfação e comprometimento afetivo medianos com o trabalho, além de percepção de sucesso na carreira. Esses seriam os possíveis motivos pelos quais os profissionais não desejam deixar a organização em que trabalham atualmente.

\subsection{CORRELAÇÕES}

Adotaram-se como parâmetros os critérios para análise de força das correlações propostos por Bisquerra, Sarriera e Martínez (2004, p. I47), a saber: $r=\mathrm{I}$, correlação perfeita; $\circ, 80<r<$ I, muito alta; $\circ, 60<r<0,80$, alta; $0,40<r<0,60$, moderada; $0,20<r<0,40$, baixa; $0<r<0,20$, muito baixa; e $r=0$, sem correlação ou nula. As mesmas interpretações serão utilizadas para os coeficientes negativos.

Os dez índices de correlação contidos na Tabela I são todos significativos, sendo seis positivos e quatro negativos. Tais resultados revelam que são diretamente proporcionais os índices de correlação entre os escores médios de satisfação no trabalho, envolvimento com o trabalho, comprometimento organizacional afetivo e percepção de sucesso na carreira, enquanto quatro indicam correlação inversamente proporcional dessas variáveis com intenção de 
rotatividade. Na Tabela I, verifica-se também que os índices de precisão são todos satisfatórios, atingindo resultados superiores a 0,70 , valor considerado por Hair, Tatham, Anderson, e Black (2005) como ponto de corte para uma medida ser considerada confiável e precisa.

Os índices com alta correlação entre si foram satisfação no trabalho, percepção de sucesso na carreira, intenção de rotatividade e comprometimento organizacional afetivo; as duas últimas também apresentaram alta correlação entre si. Segundo os mesmos critérios adotados por Bisquerra et al. (2004), verificaram-se índices moderados de correlação entre percepção de sucesso na carreira e as variáveis comprometimento organizacional afetivo e intenção de rotatividade. Além dessas variáveis, envolvimento com o trabalho obteve correlações moderadas com todas as outras variáveis do estudo.

Vale destacar que a maior correlação com intenção de rotatividade, entre as variáveis de bem-estar no trabalho, é da variável comprometimento organizacional afetivo, tornando-se assim o provável melhor preditor de intenção de rotatividade. Dessa forma, entende-se que quanto maior for o comprometimento afetivo do trabalhador com a organização, menores serão as possibilidades de ele pensar em uma eventual saída, planejá-la e manifestar vontade quanto a isso, e vice-versa.

Por fim, verifica-se que o índice de correlação entre percepção de sucesso na carreira e intenção de rotatividade é negativo. Percepção de sucesso na carreira apresenta correlação negativa, significativa e moderada com intenção de rotatividade $(r=-0,450, \mathrm{p}<0,00 \mathrm{I})$. A partir desse resultado, pode-se considerar que intenção de rotatividade, ou seja, a vontade, o pensamento e o planejamento mental organizado pelo profissional em deixar o local onde trabalha, tende a diminuir na presença de alta de percepção de sucesso na carreira, e vice-versa.

A análise dessas correlações nos mostra que, à medida que cresce a percepção de sucesso na carreira do profissional, crescem também os indicadores de bem-estar no trabalho, e, dessa forma, a intenção dos profissionais de sair das empresas em que trabalham é reduzida, pois as correlações são inversamente proporcionais. Portanto, percepção de sucesso na carreira e bem-estar no trabalho parecem ser bons indicadores de retenção profissionais, ou melhor, os profissionais com alta percepção de sucesso na carreira e que têm bem-estar no trabalho tendem a não planejar sair das empresas em que trabalham.

\subsection{ANÁLISE DE REGRESSÃO MÚLTIPLA}

Na Tabela 2, apresentam-se os resultados das análises de regressão múltipla linear (stepwise), apontando a variável dependente, os regressores, os passos em 
que eles entraram na regressão, a contribuição particular para prover explicação para a variável dependente ( $\mathrm{R}^{2}$ parcial), o total de explicação provido pelo respectivo modelo ( $\mathrm{R}^{2}$ modelo) e o coeficiente de regressão (beta padronizado), que expressa quanto cada variável independente contribuiu para a predição do critério por meio de unidades em termos de desvio padrão.

\section{TABELA 2}

\section{MODELO DE REGRESSĀO LINEAR MÚNTIPLA STEPWISE PARA INTENÇÃO DE ROTATIVIDADE}

\begin{tabular}{cccccc}
\hline $\begin{array}{c}\text { VARIÁVEL } \\
\text { DEPENDENTE }\end{array}$ & REGRESSORES & PASSOS & $\begin{array}{c}\mathrm{R}^{2} \\
\text { PARCIAIS }\end{array}$ & $\begin{array}{c}\mathrm{R}^{2} \\
\text { MODELO }\end{array}$ & BETAS PADRONIZADOS \\
\hline \multirow{2}{*}{$\begin{array}{c}\text { COA } \\
\text { Intenção de }\end{array}$} & 1 & $0,422^{* * *}$ & & $-0,363^{* * *}$ \\
rotatividade & ST & 2 & $0,039^{* * *}$ & $0,477^{* * *}$ & $-0,256^{* * *}$ \\
\cline { 2 - 6 } & ET & 3 & $0,016^{* * *}$ & & $-0,160^{* * *}$ \\
\hline
\end{tabular}

$\mathrm{COA}=$ comprometimento organizacional afetivo; $\mathrm{ST}=$ satisfação no trabalho; $\mathrm{ET}=$ envolvimento com o trabalho.

$* * * \mathrm{p}<0,001$.

Fonte: Dados da pesquisa.

Os resultados das análises de regressão linear múltipla pelo método stepwise contidas na Tabela 2 permitem identificar o impacto exercido pelos antecedentes diretos de intenção de rotatividade, que, nesse modelo, são comprometimento organizacional afetivo, satisfação no trabalho e envolvimento com o trabalho.

Deve-se ressaltar que a percepção de sucesso na carreira foi retirada pelas próprias análises do modelo de regressão, pois, por um lado, era o preditor com menor índice de correlação com intenção de rotatividade $(r=-0,450)$, tendo, por lado, correlações fortes com as três dimensões de bem-estar no trabalho, as quais foram, junto a elas, posicionadas no modelo conceitual (Figura 2) como variável independente.

A Figura 3 exibe os resultados desta pesquisa, mostrando as contribuições das variáveis antecedentes na explicação da variância da variável critério.

Dada a exclusão da percepção de sucesso na carreira das análises de regressão, pode-se afirmar que as crenças do trabalhador acerca do quanto ele alcança sucesso em sua carreira não conseguem produzir impactos significativos sobre a intenção de rotatividade. 


\section{FIGURA 3}

\section{VARIÁVEIS MANTIDAS NO MODELO, APÓS ANÁLISE DE REGRESSÃO STEPWISE}

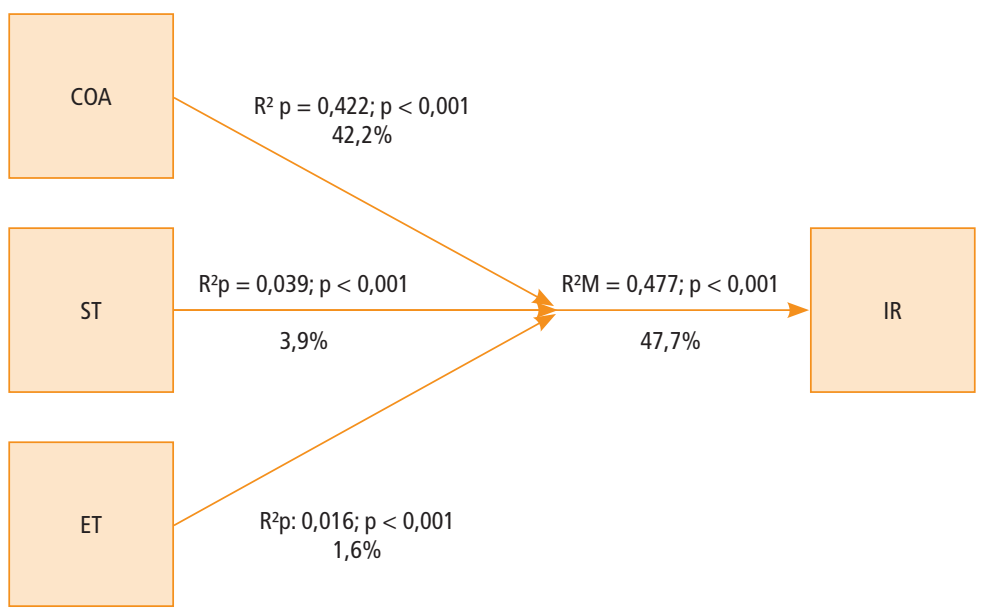

$\mathrm{COA}=$ comprometimento organizacional afetivo; $\mathrm{ST}$ = satisfação no trabalho; $\mathrm{ET}=$ envolvimento com o trabalho; IR = intenção de rotatividade.

Fonte: Elaborada pelos autores.

Esses resultados apresentados informam que o bem-estar no trabalho consegue explicar um valor próximo à metade da variabilidade de intenção de rotatividade. Assim, os planos do empregado de sair da organização recebem impacto significativo e de valor elevado do estado em que ele se encontra quanto à sua sensação de bem-estar.

Ainda se pode ressaltar que, dentre as três dimensões de bem-estar no trabalho, a que mais exerce impacto sobre a intenção de rotatividade é o compromisso afetivo que o empregado nutre pela empresa em que trabalha. Pelos valores dos betas padronizados contidos na Tabela 2 , fica evidente que o comprometimento afetivo com a empresa ultrapassa os valores de satisfação no trabalho e envolvimento com o trabalho, tornando o componente mais importante de bem-estar no trabalho na predição de intenção de rotatividade, corroborando os estudos já realizados (Polizzi, 20II; Suliman \& Al-Junaibi, 20ıо).

\section{CONSIDERAÇÕES FINAIS}

Os resultados permitem concluir que este estudo traz importantes contribuições para a prática organizacional, tornando evidente que, para as empresas 
contarem com uma força de trabalho estável, é essencial que os gestores se preocupem em manter seus colaboradores satisfeitos em relação à chefia, aos colegas, ao trabalho em si, ao salário e às promoções. E as organizações precisam buscar artifícios para que seus colaboradores se mantenham envolvidos com o trabalho e, sobretudo, comprometidos afetivamente com a empresa, ou seja, elas devem se preocuparem em tornar o ambiente empresarial agradável e estimulante, com perspectivas animadoras em relação ao futuro dos seus trabalhadores. Assim, poderão fazer com que a intenção de seus profissionais em abandoná-la seja em muito reduzida, particularmente diante de um cenário em que o cuidado com as pessoas no ambiente de trabalho tornou-se fator importante por agregar valor a qualquer tipo de negócio.

Os resultados evidenciaram que os trabalhadores estudados parecem estar parcialmente satisfeitos com os colegas de trabalho, a chefia, o salário, as oportunidades de serem promovidos na empresa e também com o quanto suas tarefas são capazes de absorvê-los. No entanto, sobre o envolvimento dos trabalhadores pesquisados, eles tendem a discordar que as maiores satisfações de suas vidas vêm do seu trabalho, apesar de demonstrarem-se parcialmente comprometidos afetivamente com a organização, segundo os resultados das análises.

Quanto à percepção de sucesso na carreira, os participantes desta pesquisa não estão muito confiantes a respeito de suas realizações acumuladas como resultado de sua experiência de trabalho. No entanto, em relação à intenção de rotatividade, esses mesmos profissionais, cujas percepções de sucesso estão relativamente baixas, não apresentam planos, pensamentos e vontade de deixar o emprego atual, de acordo com os resultados alcançados.

A intenção de rotatividade dos trabalhadores pesquisados é fortemente influenciada pelo comprometimento organizacional afetivo deles para com a empresa em que trabalham. Dessa forma, pode-se concluir que quanto mais a empresa leva o indivíduo a se sentir animado, interessado e entusiasmado por trabalhar para ela, menos os profissionais pensarão em deixá-la.

Na literatura nacional, surge o conceito de percepção de sucesso na carreira que necessita de mais estudos, principalmente na relação com outras variáveis do comportamento organizacional. Nesse contexto, podem-se buscar, em futuras investigações, novas variáveis que possam atuar como preditoras de intenção de rotatividade, bem como identificar variáveis do comportamento organizacional que tenham capacidade para atuar com a percepção de sucesso na carreira como independentes em um modelo.

Finalizando, entre as práticas organizacionais, podemos citar o cuidado que a empresa deve ter com o cliente interno, remuneração compatível com o mercado, realização de avaliações de desempenho coerentes, política atraente de cargos 
e salários, treinamento, desenvolvimento e planos de sucessão adequados, criação de um ambiente que estimule a inovação e que fomente um ambiente sem medo de represálias. Dessa forma, existe maior probabilidade de produzirem resultados satisfatórios e eficazes, contribuindo para a competitividade de sua organização empregadora no mercado.

Este trabalho teve como objetivo maior contribuir para os estudos da área de gestão de pessoas, ampliando os conhecimentos sobre o fenômeno intenção de rotatividade. E, por fim, pesquisas futuras na investigação do impacto exercido por percepções de sucesso na carreira e por bem-estar no trabalho sobre intenção de rotatividade campo do comportamento organizacional podem sugerir novas linhas de pesquisa.

\section{WELL-BEING AT WORK AND PERCEPTION OF CAREER SUCCESS AS ANTECEDENT FOR TURNOVER INTENTION}

\section{ABSTRACT}

The aim of this study was to analyze, interpret and discuss the relationship between perceptions of career success, well-being at work and turnover intention of workers in Southeast Brazil. The study gathered 500 employees working in the state of Sao Paulo in non-governmental, public and private organizations. For data collection, an auto-filling questionnaire composed by five scales which measured the research variance. The work aimed to present, interpret and discuss the relations among variances, as well as to test hypotheses regarding the proposed conceptual model, by means of a transversal research in the quantitative approach. The collected data were analyzed by applying parametric statistical techniques (calculation of descriptive statistics: means, standard deviation, T test and correlations; multivariate statistic calculations; analysis of and stepwise multiple linear regression and test of normality of the variables, using the Kolmogorov-Smirnov test). Data treatment and analysis were performed by the statistical software Statistical Package for Social Sciences - SPSS version I8.o. The results showed that the dimensions of well-being at work have a strong and significant impact on turnover intention of professionals, while the perception of career success has contributed to low values in this impact, due to the shape of the hypothetical model. The research led us to conclude that the more the company is concerned with providing an exciting, interesting, and enthusiastic work environment, the fewer professionals consider leaving it. 


\section{KEYWORDS}

Organizational behavior. Well-being at work. Perception of career success. Career. Turnover.

\section{BIENESTAR EN EL TRABAJO Y LA PERCEPCIÓN DE ÉXITO EN LA CARRERA COMO ANTECEDENTES DE LA INTENCIÓN DE ROTACIÓN DE PERSONAL}

\section{RESUMEN}

El objetivo de este estudio fue analizar, interpretar y discutir la relación entre la percepción de éxito profesional, el bienestar en el trabajo y la intención de rotación entre los trabajadores en el sureste de Brasil. Participaron de esta investigación 500 trabajadores que actúan en el estado de São Paulo en organizaciones no gubernamentales, públicas y privadas. Como instrumento para recolectar los datos se utilizó un cuestionario de auto relleno compuesto de cinco escalas que midieron las variables de la investigación. La presente investigación se propuso presentar, interpretar y discutir las relaciones entre las variables, como también, testar las hipótesis que se refieren al modelo conceptual propuesto, mediante una investigación de naturaleza transversal con abordaje cuantitativo, cuyos datos recolectados fueron analizados por aplicación de técnicas estadísticas paramétricas (cálculos de estadísticas descriptivas: promedios, desvío patrón, teste $t$ y correlaciones; cálculos de estadísticas multivariadas: análisis de regresión linear múltiple stepwise y teste de normalidad de las variables, mediante el teste de Kolmogorov-Smirnov). El tratamiento y el análisis se realizaron con el software Statistical Package for Social Sciences - SPSS, versión I8.o. Los resultados mostraron que el bienestar en las dimensiones del trabajo tiene un impacto fuerte y significativo sobre la intención de la rotatividad de los profesionales, mientras que la percepción de éxito profesional contribuyó con valores bajos en este impacto debido al formato del modelo hipotético. La investigación nos llevó a la conclusión de que cuanto más la empresa se ocupa de proporcionar un ambiente de trabajo que sea emocionante, interesante y que provoca entusiasmo, menos profesionales pensarán en dejarla.

\section{PALABRAS CLAVE}

Comportamiento organizacional. Bienestar en el trabajo. Percepción de éxito profesional. Carrera. Rotación de personal. 


\section{REFERÊNCIAS}

Albuquerque, A. S., \& Tróccoli, B. T. (2004). Desenvolvimento de uma escala de bem-estar subjetivo. Psicologia: Teoria e Pesquisa, 20, I53-I64.

Bisquerra, R., Sarriera, J. C., \& Martínez, F. (2004). Introdução à estatística: enfoque informático com o pacote estatístico SPSS. Porto Alegre: Artmed.

Brayfield, A. H., \& Crockett, W. H. (I955). Employee attitudes and employee performance. Psychological Bulletin, 52, 396-424.

Brief, A., \& Weiss, H. (2002). Organizational behavior: affect in the workplace. Annual Review of Psychology, 53, 279-307.

Busbin, J. W., Johnson, J. T., \& DeConinck, J. (2008). The evolution of sustainable competitive advantage: from value chain to modular outsource networking. Competition Forum, 6, I03-I08.

Campos, C. A., \& Malik, A. M. (2008). Satisfação no trabalho e rotatividade dos médicos do programa de saúde da família. Revista de Administração Pública, 42, 347-368.

Cavazotte, F. S. C. N., Oliveira, L. B., \& Miranda, L. C. (20I0). Desigualdade de gênero no trabalho: reflexos nas atitudes das mulheres e em sua intenção de deixar a empresa. Revista de Administração, $45,70-83$.

Costa, L. (20II). A relação entre a percepção de sucesso na carreira e o comprometimento organizacional: um estudo entre professores de universidades privadas selecionadas da grande São Paulo. Tese de doutorado, Universidade de São Paulo, São Paulo, SP, Brasil.

Csikszentmihalyi, M. (I999). A descoberta do fluxo: a psicologia do envolvimento com a vida cotidiana. Rio de Janeiro: Rocco.

Csikszentmihalyi, M. (2003). Gestão qualificada: a conexão entre felicidade e negócio. Porto Alegre: Artmed.

Daniels, K. (2000). Measures of five aspects of affective well-being at work. Human Relations, 53, 275-294.

Davis, K., \& Newstrom, J. W. (2002). Comportamento humano no trabalho: uma abordagem psicológica. São Paulo: Pioneira.

Dessen, M. C., \& Paz, M. G. T. (20I0). Validação do instrumento de indicadores de bem-estar pessoal nas organizações. Psicologia em Estudo, 15, 409-4I8.

Diener, E. (1984). Subjective well-being. Psychological Bulletin, 95, 542-575.

DiMaggio, P. J., \& Powell, W. W. (I983). The iron cage revisited: institutional isomorphism and collective rationality in organizational fields. American Sociological Review, 48, I47-160.

Dutra, J. S. (2002). Gestão de pessoas: modelo, processos, tendências e perspectivas. São Paulo: Atlas.

Ferreira, M. L. C. B. (200I). Antecedentes de intenção de rotatividade: estudo de um modelo psicossocial. Dissertação de mestrado, Universidade Metodista de São Paulo, São Bernardo do Campo, SP, Brasil. Gondim, S. M. G., \& Siqueira, M. M. M. (2004). Emoções e afetos no trabalho. In J. C. Zanelli, J. E. Borges-Andrade \& A. B. Bastos (Orgs). Psicologia, organizações e trabalho no Brasil (pp. 207-236). Porto Alegre: Artmed.

Gubman, E. L. (I998). The talent solution: aligning strategy and people to achieve extraordinary results. New York: McGraw-Hill.

Hair, J. F., Tatham, R. L., Anderson, R. E., \& Black, W. (2005). Análise multivariada de dados. Porto Alegre: Bookman. 
Herzberg, F., Mausner, B., Peterson, R. O., \& Capwell, D. F. (I959). Job attitudes: review of research and opinion. Industrial and Labor Relations Review, 12, 320-322.

Hughes, E. C. (I937). Institutional office and the person. American Journal of Sociology, 43, 404-4I3. Le Boterf, G. (2003). Desenvolvendo a competência dos profissionais. Porto Alegre: Bookman Artmed. Lodahl, T. M., \& Kejner, M. (1965). The definition and measurement of job involvement. Journal of Applied Psychology, 49, 23-33.

London, M., \& Stumpf, S. A. (I982). Managing careers. Virginia: Addison Wesley Publishing Company. Maynard, D. C., \& Joseph, T. A. (2008). Are all part-time faculty underemployed? The influence of faculty status preference on satisfaction and commitment. Higher Education, 55, I35-I54.

Medeiros, C. R. G., Junqueira, A. G. W., Schwingel, G., Carreno, I., Jungles, L. A., \& Saldanha, O. M. F. L. (20IO). A rotatividade de enfermeiros e médicos: um impasse na implementação da Estratégia de Saúde da Família. Ciência \& Saúde Coletiva, 15, I52I-I53I.

Menezes, I. G. (2009). Comprometimento organizacional: construindo um conceito que integre atitudes e intenções organizacionais. Tese de doutorado, Universidade Federal da Bahia, Salvador, BA, Brasil. Menezes, I. G., \& Bastos, A. B. (20I0). Propriedades psicométricas da escala de intenções comportamentais de permanência na organização (EICPO). Estudos e Pesquisas em Psicologia, 3, 800-8I7. Mintzberg, H., \& Lampel, J. (I999). Reflecting on the strategy process. Sloam Management Review, 40(3), 2I-30.

Motta, R. (2006). Reflexões sobre a customização das carreiras gerenciais: a individualidade e a competitividade contemporâneas. In M. Balassiano \& I. Costa (Orgs.). Gestão de carreiras: dilemas e perspectivas. São Paulo: Atlas.

Mowday, R. T., Steers, R. M., \& Porter, L. W. (I979). The measurement of organizational commitment. Journal of Vocational Behavior, 14, 224-247.

Nunnally, J. C. (I975). Introductory statistics for psychology and education. New York: McGraw-Hill. Paschoal. T., \& Tamayo, A. (2008). Construção e validação da escala de bem-estar no trabalho. Avaliação Psicológica, 7, II-22.

Polizzi Filho, A. (20II). O impacto de bem-estar no trabalho e de capital psicológico sobre intenção de rotatividade: um estudo com professores. Dissertação de mestrado, Universidade Metodista de São Paulo, São Bernardo do Campo, SP, Brasil.

Ryff, C. D., \& Keyes, C. L. M. (I998). The structure of psychological well-being revisited. Journal of Personality and Social Psychology, 69, 719-727.

Siqueira, M. M. M. (I995). Antecedentes de comportamentos de cidadania organizacional: análise de um modelo pós-cognitivo. Tese de doutorado, Universidade de Brasília, Brasília, DF, Brasil.

Siqueira, M. M. M. (2008). Medidas do comportamento organizacional: ferramentas de diagnóstico e de gestão. Porto Alegre: Artmed.

Siqueira, M. M. M. (2009). Bem-estar no trabalho. In J. Cruz, S. N. Jesus \& C. Nunes (Org.). Bem-estar e qualidade de vida: contributos da psicologia da saúde (pp. 249-264). Leiria: Textiverso.

Siqueira, M. M. M., \& Gomide, S., Jr. (2004). Vínculos do indivíduo com o trabalho e com a organização. In J. C. Zanelli, J. E. Borges-Andrade \& A. B. Bastos (Orgs.). Psicologia, organizações e trabalho no Brasil (pp. 300-328). Porto Alegre: Artmed.

Siqueira, M. M. M., Gomide, S., Jr., Moura, O. I., \& Marques, T. M. (I997). Um modelo pós-cognitivo para intenção de rotatividade: antecedentes afetivos e cognitivos. Resumo do Congresso Interamericano de Psicologia, São Paulo, SP, Brasil, 26. 
Siqueira, M. M. M., \& Padovam, A. R. (2008). Bases teóricas de bem-estar subjetivo, bem-estar psicológico e bem-estar no trabalho. Psicologia: Teoria e Pesquisa, 24, 20I-209.

Stewart, R., Volpone, S., Avery, D., \& McKay, P. (20II). You support effects of diversity and ethical climate perceptions on turnover intentions. Journal of Business Ethics, 100, 58I-593.

Suliman, A. A., \& Al-Junaibi, Y. (20I0). Commitment and turnover intention in the UAE oil industry. The International Journal of Human Resource Management, 21, I472-1489.

Tamayo, A. (2000). Prioridades axiológicas e satisfação no trabalho. Revista de Administração, 35, 37-47. Van Horn, J. E., Taris, T. W., Schaufeli, W. B., \& Scheurs, J. G. (2004). The structure of occupational well-being: a study among Dutch teachers. Journal of Occupational and Organizational Psychology, 77, 365-375.

Vroom, H. (1964). Work and motivation. New York: Wiley.

Watson, D., Clark, L. A., \& Tellegen, A. (I988). Development and validation of brief measures of positive and negative affect: the PANAS scales. Journal of Personality and Social Psychology, 54, $1063-1067$.

\section{APÊNDICE}

\section{INSTRUMENTO DE COLETA DE DADOS}

Esta pesquisa tem por finalidade coletar dados para um estudo sobre o que os trabalhadores pensam a respeito do seu trabalho, da sua carreira e da empresa onde atuam.

Abaixo, estão listados vários sentimentos que alguém poderia ter em relação à empresa onde trabalha. Gostaríamos de saber o quanto você sente estes sentimentos. Dê suas respostas anotando, nos parênteses que antecedem cada frase, aquele número (de i a 5) que melhor representa sua resposta.

\begin{tabular}{c|c|c|c|c}
\hline Nada & Pouco & Mais ou menos & Muito & Extremamente \\
\hline 1 & 2 & 3 & 4 & 5 \\
\hline
\end{tabular}

A empresa onde trabalho faz-me sentir...

I. ( ) Orgulhoso dela.

2. ( ) Contente com ela.

3. ( ) Entusiasmado com ela.
4. ( ) Interessado por ela.

5. ( ) Animado com ela.

As frases a seguir falam a respeito de alguns aspectos do seu trabalho atual. Indique o quanto você se sente satisfeito ou insatisfeito com cada um deles. Dê suas 
respostas anotando, nos parênteses que antecedem cada frase, aquele número (de I a 7) que melhor representa sua resposta.

\begin{tabular}{c|c|c|c|c|c|c}
\hline $\begin{array}{c}\text { Totalmente } \\
\text { insatisfeito }\end{array}$ & $\begin{array}{c}\text { Muito } \\
\text { insatisfeito }\end{array}$ & Insatisfeito & Indiferente & Satisfeito & $\begin{array}{c}\text { Muito } \\
\text { satisfeito }\end{array}$ & $\begin{array}{c}\text { Totalmente } \\
\text { satisfeito }\end{array}$ \\
\hline 1 & 2 & 3 & 4 & 5 & 6 & 7 \\
\hline
\end{tabular}

No meu trabalho atual sinto-me...

6. ( ) Com o espírito de colaboração dos meus colegas de trabalho.

7. ( ) Com o número de vezes que já fui promovido nessa empresa.

8. ( ) Com o meu salário comparado com o quanto eu trabalho.

9. ( ) Com o tipo de amizade que meus colegas demonstram por mim.

Io. ( ) Com o grau de interesse que minhas tarefas me despertam.

II. ( ) Com o meu salário comparado à minha capacidade profissional.

I2. ( ) Com a maneira como esta empresa realiza promoções de seu pessoal.

I3. ( ) Com a capacidade do meu trabalho absorver-me.

I4. ( ) Com as oportunidades de ser promovido nessa empresa.

I5. ( ) Com o entendimento entre mim e meu chefe.

I6. ( ) Com meu salário comparado aos meus esforços no trabalho.

I7. ( ) Com a maneira como meu chefe me trata.

I8. ( ) Com a variedade de tarefas que realizo.

I9. ( ) Com a confiança que eu posso ter em meus colegas de trabalho.

20. ( ) Com a capacidade profissional do meu chefe.

A seguir estão cinco frases referentes ao seu trabalho atual. Indique o quanto você concorda ou discorda de cada uma delas. Dê suas respostas anotando, nos parênteses que antecedem cada frase, aquele número (de I a 7), que melhor representa sua resposta.

\begin{tabular}{c|c|c|c|c|c|c}
\hline $\begin{array}{c}\text { Discordo } \\
\text { totalmente }\end{array}$ & $\begin{array}{c}\text { Discordo } \\
\text { moderadamente }\end{array}$ & $\begin{array}{c}\text { Discordo } \\
\text { levemente }\end{array}$ & $\begin{array}{c}\text { Nem discordo, } \\
\text { nem concordo }\end{array}$ & $\begin{array}{c}\text { Concordo } \\
\text { levemente }\end{array}$ & $\begin{array}{c}\text { Concordo } \\
\text { moderadamente }\end{array}$ & $\begin{array}{c}\text { Concordo } \\
\text { totalmente }\end{array}$ \\
\hline 1 & 2 & 3 & 4 & 5 & 6 & 7 \\
\hline
\end{tabular}

2I. ( ) As maiores satisfações de minha vida vêm do meu trabalho.

22. ( ) As horas que passo trabalhando são as melhores horas do meu dia.

23. ( ) As coisas mais importantes que acontecem em minha vida envolvem meu trabalho. 
24. ( ) Eu como, vivo e respiro o meu trabalho.

25. ( ) Eu estou pessoalmente muito ligado ao meu trabalho.

A seguir estão três frases que podem representar alguns pensamentos seus. Dê suas respostas anotando, nos parênteses que antecedem cada frase, aquele número (de i a 5) que melhor representa sua resposta.

\begin{tabular}{c|c|c|c|c}
\hline Nunca & Raramente & Às vezes & Frequentemente & Sempre \\
\hline 1 & 2 & 3 & 4 & 5 \\
\hline
\end{tabular}

26. ( ) Penso em sair da empresa onde trabalho.

27. ( ) Planejo sair da empresa onde trabalho.

28. ( ) Tenho vontade de sair da empresa onde trabalho.

As próximas questões referem-se a toda a sua carreira profissional. Indique o quanto você concorda ou discorda de cada uma delas. Dê suas respostas anotando, nos parênteses que antecedem cada frase, aquele número (de i a 7), que melhor representa sua resposta.

\begin{tabular}{c|c|c|c|c|c|c}
\hline $\begin{array}{c}\text { Discordo } \\
\text { totalmente }\end{array}$ & $\begin{array}{c}\text { Discordo } \\
\text { moderadamente }\end{array}$ & $\begin{array}{c}\text { Discordo } \\
\text { levemente }\end{array}$ & $\begin{array}{c}\text { Nem discordo, } \\
\text { nem concordo }\end{array}$ & $\begin{array}{c}\text { Concordo } \\
\text { levemente }\end{array}$ & $\begin{array}{c}\text { Concordo } \\
\text { moderadamente }\end{array}$ & $\begin{array}{c}\text { Concordo } \\
\text { totalmente }\end{array}$ \\
\hline 1 & 2 & 3 & 4 & 5 & 6 & 7 \\
\hline
\end{tabular}

29. ( ) Tenho orgulho do que faço profissionalmente.

30. ( ) Estou tranquilo sobre meu futuro quanto às minhas necessidades financeiras e materiais.

3I. ( ) Estou constantemente aprendendo e me desenvolvendo em minha carreira.

32. ( ) Tenho criado inovações importantes durante minha carreira profissional.

33. ( ) Tenho uma boa rede de contatos profissionais.

34. ( ) Minha carreira é compatível com minhas vocações.

35. ( ) Tenho sucesso nas avaliações da minha competência.

36. ( ) Meu trabalho gera efeitos positivos na sociedade.

37. ( ) Tenho alcançado minhas metas de nível hierárquico (ou cargos) em minha carreira.

38. ( ) Sou reconhecido(a) pelos colegas de trabalho.

39. ( ) O prestígio (ou status) profissional da minha posição hierárquica está de acordo com meus interesses.

40. ( ) Minha renda supre as necessidades minhas e de meus dependentes. 


\section{DADOS COMPLEMETARES}

4I. Sexo:
( ) Masculino
( ) Feminino

42. Idade: anos.

43. Estado civil:
( ) solteiro(a) ou separado(a)
( ) casado(a) ou amasiado(a)
( ) outros

44. Grau de instrução:
( ) Ensino fundamental incompleto
( ) Ensino fundamental completo
( ) Ensino médio incompleto
( ) Ensino médio completo
( ) Superior incompleto
( ) Superior completo
( ) Pós-graduação incompleta
( ) Pós-graduação completa

45. Tempo de trabalho nessa empresa: anos.

Se for menos de um ano assinale aqui: ( ) 